\title{
EXPLORATION OF WHOLE ATMOSPHERE LidAR: MACH-ZEHNDER RECEIVER TO EXTEND FE DOPPLER LIDAR WIND MEASUREMENTS FROM THE THERMOSPHERE TO THE GROUND John A. Smith ${ }^{1 *}$ and Xinzhao Chu ${ }^{1}$ \\ ${ }^{1}$ University of Colorado Boulder, 216 UCB, CIRES, Boulder, CO 80309, USA, \\ *Email:John.A.Smith@,colorado.edu andXinzhao.Chu@colorado.edu
}

\begin{abstract}
A receiver employing a field-widened Mach-Zehnder interferometer (MZI) is investigated for extending the wind measurement range of a narrowband Fe Doppler $(372 \mathrm{~nm})$ lidar from its existing measurement range in the mesosphere and lower thermosphere (MLT) down to the ground. This design uses the multiple transmitted frequencies available from the base Fe Doppler lidar in combination with an MZI receiver to make a measurement of the Doppler shift from Rayleigh-Mie scattering that is independent of aerosol backscatter ratio, temperature and pressure of the lidar volume and also independent of geometric overlap, the chopper function and any other factor affecting the signal in both MZI channels equally. A ratio is constructed from the three frequencies and two channels of the interferometer that exhibits a measurement performance of 1.75 times the Cramer-Rao lower bound, which is comparable to the dual MZI (DMZ) while preserving the insensitivity to backscatter spectrum of the quad MZI (QMZ). Using actual data obtained recently from the Fe Doppler lidar, we show the expected measurement performance of this whole atmosphere lidar instrument concept.
\end{abstract}

\section{INTRODUCTION}

The Fe Doppler lidar is one of a class of lidars referred to as "resonance Doppler" lidars that exploit the exceptionally high backscatter cross-section of metal atoms deposited by meteor ablation in the MLT region, typically $\mathrm{Fe}, \mathrm{Na}$ or $\mathrm{K}$, to sense wind and temperature in this region. By probing the backscatter cross-section at three fixed frequencies distributed about the resonance line, these lidars infer the line's Doppler broadening and bulk Doppler shift, thereby making measurements of the temperature and wind of the backscatter volume, respectively [1]. In light of the recent discoveries of neutral metal layers well into the thermosphere of 100 $200 \mathrm{~km}$ [2], the Fe Doppler lidar is now thought to be capable of measuring wind and temperature in the 75 to $\sim 200 \mathrm{~km}$ region.

To extend resonance Doppler lidar wind measurements to the lower mesosphere and stratosphere, we propose the addition of a Mach-Zehnder interferometer as a spectral discriminator in the receiver system of the $\mathrm{Fe}$ Doppler lidar to discern Doppler shifts from Rayleigh and Mie scattering (see Figure 1). An Fe Doppler lidar equipped with such an instrument would be especially well-suited to fulfilling the need for wind measurement in the so-called "radar gap" in the statically stable region 30-60 km, which lacks sufficient turbulence or ionization for routine radar measurement. Therefore, a mobile "whole-atmosphere" lidar that implements such a receiver could present a much-needed capability to the atmospheric science community. Despite the potential scientific benefits, routine wind profiling through the complete atmospheric column remains elusive for a single system and usually requires campaigns spanning multiple earth observing platforms incorporating balloons, MST radars and rockets. For example, the ability to measure wind and temperature from 35 to $150+\mathrm{km}$ with a single, mobile lidar would help to discover the source(s) of short-period inertia gravity waves recently observed in Antarctica [2], [3].

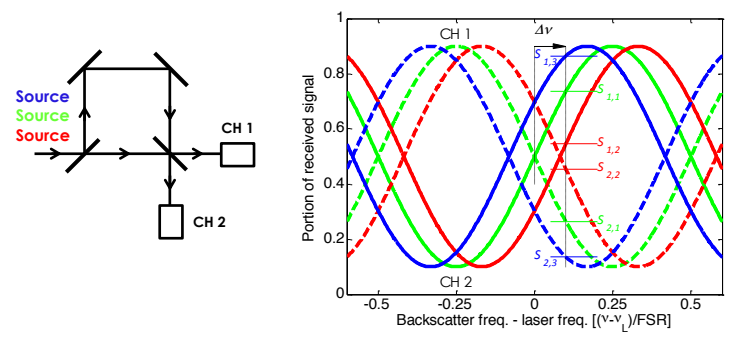

Figure 1. Response functions of the XMZ apparatus introduced in this work. Signals from all channels and frequencies exhibit sensitivity to the Doppler shift.

Atomic line filters have been implemented in $\mathrm{Na}$ Doppler lidars [4], [5] to infer wind from Rayleigh scattering, but the low power attainable from current $589 \mathrm{~nm}$ transmitters and relatively weak molecular scattering at this wavelength severely limits the attainable precision of wind and temperature in the radar gap. Fe lidars, by contrast, operating at $372 \mathrm{~nm}$ with higher power transmitters are much more capable in the Rayleigh region and can attain a comparable signal in the MLT region due to the much higher abundance of $\mathrm{Fe}$ compared to $\mathrm{Na}$ [1].

Liu and Kobayashi [6] were the first to propose the use of a Mach-Zehnder interferometer (MZI) as a frequency discriminator for direct-detection Doppler 
lidar. Their proposed transmitter employed the $\mathrm{Nd}$ :YAG fundamental $(1.064 \mu \mathrm{m})$ and inferred wind predominantly from aerosol, rather than molecular scattering. Bruneau [7] optimized their MZI for molecular scattering and demonstrated wind measurements up to $40 \mathrm{~km}$ [8]. Our approach is based on the MZI tuned to molecular scattering since our interests are predominantly in the stratosphere and higher where scattering from aerosols, which are relatively sparse in this region, is much weaker than that from molecules. In addition, the shorter optical path difference of an MZI optimized for molecular scattering, versus an MZI optimized for aerosol scattering, allows more extensive field-widening [9], which is crucial for resonance lidars that require a large transmitter beam divergence to avoid saturation of the layer and large collecting areas to detect single photon signal levels from the upper atmospheres [10].

\section{METHODOLOGY}

Fe lidars are unique in that the high backscatter crosssection of molecules at the $\mathrm{UV} F$ transition wavelength, and typically high power of lasers at this wavelength, allows them to serve as highly effective Rayleigh lidars also. Laser technology at the time limited the first Fe lidars to broadband systems that were capable only of temperature measurement. Recent developments have introduced narrowband systems capable of measuring wind also, the Fe-Doppler lidar at Boulder, Colorado [11] being the first example of such a lidar. This lidar is capable of measuring temperature and wind in the MLT region and temperature below the MLT region down to $30 \mathrm{~km}$ with a transmitter locked to the $\mathrm{Fe} \mathrm{a} \mathrm{a}^{5} \mathrm{D}_{4}-\mathrm{z}^{5} \mathrm{~F}_{5}$ transition at $372.0995 \mathrm{~nm}$ and shifted alternately in time by $742 \mathrm{MHz}$ above and below the precise transition wavelength. Its reliance on spectroscopy to derive spectral information from species in the MLT region restricts the routine wind measurement range to regions of persistent $\mathrm{Fe}$, or about $75-115 \mathrm{~km}$. Therefore, to measure wind below this range requires a spectrally sensitive receiver.

In this work, we analyze a field-widened dual MachZehnder (DMZ) for the task of combining, using the same resonance Fe-Doppler beam, Rayleigh Doppler lidar measurements from the troposphere to mesosphere with resonance Doppler measurements that extend upwards from the mesosphere to the lower thermosphere. The concept is then to marry a RayleighMie Doppler direct-detection lidar technique with the resonance Doppler lidar technique to cover wind measurement throughout the whole atmosphere. Rather than operating at a single transmitted frequency, as previous works [6], [7] have investigated, we investigate the benefits of using the three available transmitted frequencies of this resonance Doppler lidar system in combination with the DMZ system. We will refer to this scheme as the $\mathrm{X}$-frequency Mach-Zehnder or "XMZ" since, in principle, an arbitrary number of spectral response phases/frequencies is possible in this scheme (Error! Reference source not found.). By using the information from three frequencies, a Doppler shift estimate can be made independent of the atmospheric contrast factor in addition to instrument defects. The benefits of the XMZ technique are possible due to a reduction in the number of variables compared to the QMZ technique (Figure) presented in previous works [6], [7], which required a waveplate, two polarizers and two additional detectors, each with associated uncertainties.

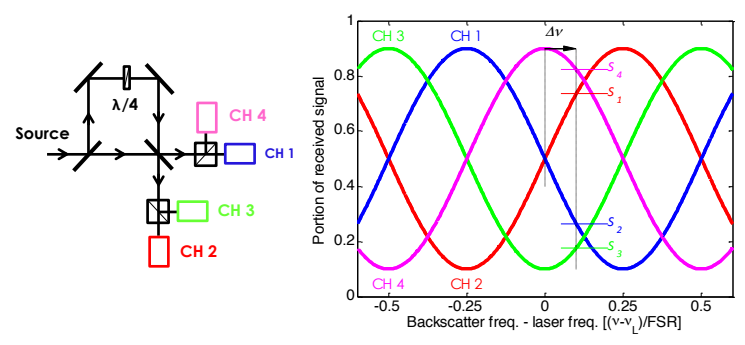

Figure 2. Response functions of the QMZ apparatus introduced by previous authors. Two of the four channels are insensitive to Doppler shift.

Another key benefit of the Mach-Zehnder techniques, including the $\mathrm{XMZ}$ technique described here, is that all of the signal is recovered and summation of the signals in both channels results in a quantity free of interference effects, provided the detectors are balanced. Therefore, after summation, the same resonance Doppler lidar techniques for retrieving wind and temperature in the MLT region are still valid. Unlike many other techniques that may discard a portion of the backscatter [4], [5], [12], the MZI techniques, by recovering the complete spectrum, enable a single receiver to profile wind and temperature through the complete atmospheric column with nearly the same precision as the original instrument and without modification to any existing retrieval techniques.

\section{RESULTS}

An excellent treatment of the theory of the measurement of Doppler shift using the DMZ and QMZ techniques is provided in Bruneau [7]. Discriminator signals for the $\mathrm{XMZ}$ and $\mathrm{QMZ}$ configurations can be defined that are both insensitive to temperature, aerosol and pressure variations of the lidar volume. The XMZ and QMZ configurations are therefore our preferred methods for retrieval of wind in the stratosphere and upper troposphere. One additional 
advantage of the XMZ configuration is that since all three frequencies and associated discriminator signals are sensitive to the Doppler shift, one can then define a discriminator signal that yields a measurement performance similar to the DMZ, but, unlike the DMZ, is insensitive to backscatter spectrum. The shot-noise limited performance of the XMZ, DMZ and QMZ techniques is compared here.

The frequency of the AOM shift is $742 \mathrm{MHz}$, reference temperature is $250 \mathrm{~K}$ and the wavelength is $372 \mathrm{~nm}$. The measurement performance of all three techniques is given here, with performance for the $\mathrm{DMZ}$ and QMZ techniques given by Bruneau [7] (1). We assume that the only source of noise is shot noise and that the background is negligible.

$$
\begin{aligned}
\Delta u_{Q M Z}^{\prime}=\Delta Q \cdot\left(\frac{d Q}{d u^{\prime}}\right)^{-1} & \sim \frac{2.33}{\sqrt{\sum_{i} S_{i}}} \\
\Delta u_{D M Z}^{\prime} & \sim \frac{1.65}{\sqrt{\sum_{i} S_{i}}} \\
\Delta u_{X M Z}^{\prime} & \sim \frac{1.75}{\sqrt{\sum_{i} S_{i}}}
\end{aligned}
$$

The XMZ possesses a measurement performance that is $25 \%$ better than the QMZ while preserving the QMZ's key feature, which is the insensitivity to aerosol, temperature and pressure effects.

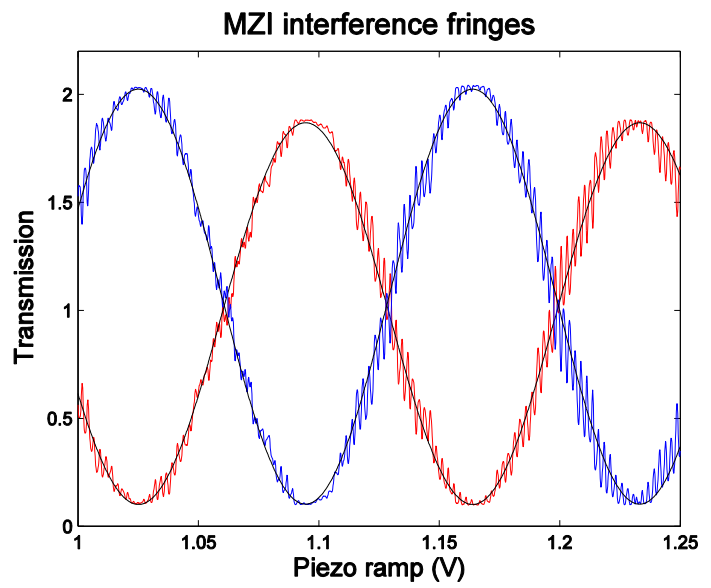

Figure 3. Mach-Zehnder fringes in both channels with sinusoidal fits indicated by the black curves to determine the instrumental contrast coefficients.

Field-widening using the compensating plate has been demonstrated at the University of Colorado Boulder and an instrument-limited fringe contrast of between 0.89 and 0.93 has been obtained with the apparatus (Figure). One leg of the interferometer is varied by ramping a piezo-actuated stage to view the interference. The mirror, beamsplitters and field compensating plate were polished and coated by
LightMachinery to obtain transmitted and reflected wavefront errors of better than $\lambda / 30$. A fiber with a core diameter of $1000 \mu \mathrm{m}$ and NA of 0.22 was filled on the input side with light from a narrow linewidth, Toptica DL-100 372-nm external cavity diode laser. Illumination from this fiber provided the range of angles representing the optical extent of the true receiver source. Frequencies matching resonant acoustic modes of the enclosure were evident in the scans from insufficient damping of ambient acoustic noise, but the long-term drift is small due to the thermally compensated nature of this design.

Based on data obtained from the Fe Doppler lidar to date, we have simulated the uncertainties. This figure includes data that was taken with the transmitter operating at a reduced power level of $1.45 \mathrm{~W}$ due to optical damage concerns. An upgrade to the optics will allow the lidar to operate at power levels of up to $6 \mathrm{~W}$. Once the design power is attained, the wind measurement precision will be within $4 \mathrm{~m} / \mathrm{s}$ for 15 minute and $3 \mathrm{~km}$ resolutions up to $75 \mathrm{~km}$, above which the wind measurement is accomplished from the resonance 3-frequency technique. For the present power levels, we still expect a precision of $1 \mathrm{~m} / \mathrm{s}$ up to $50 \mathrm{~km}$ and a maximum uncertainty of $8 \mathrm{~m} / \mathrm{s}$ at $76 \mathrm{~km}$ at a resolution of 15 minutes horizontally and $2.9 \mathrm{~km}$ vertically (Figure 4). The parameters of the Fe Doppler lidar transmitter and receiver are listed in Error! Reference source not found..

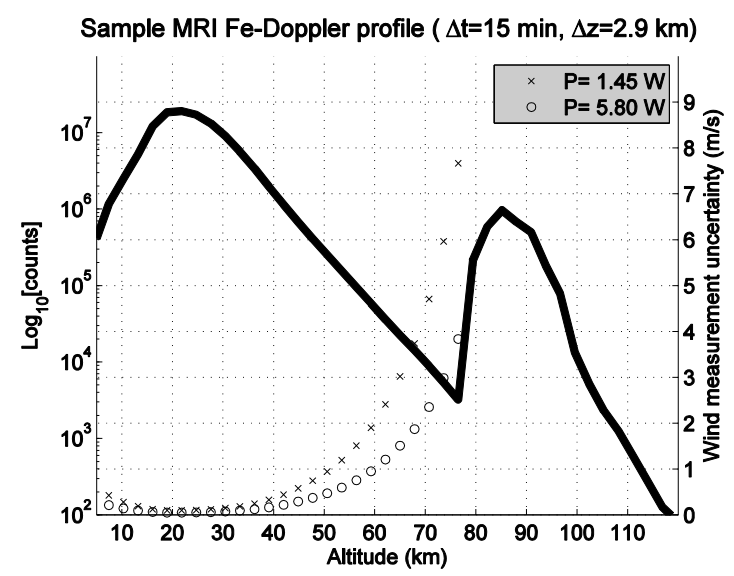

Figure 4. Based on data obtained from the Fe-Doppler lidar to date, we predict the line-of-sight wind measurement uncertainty.

\section{CONCLUSIONS}

We have described a method of extending the wind measurement range of an Fe-Doppler lidar from the MLT region down to the surface or near surface using a tilted, field-widened Mach-Zehnder interferometer. By detecting the signal at both channels of the interferometer, and at three distinct frequencies within 
a quarter of the free spectral range, the measurements can be made independent of the aerosol backscatter ratio, temperature and Brillouin scattering contributions to the backscatter spectrum. In addition, the uncertainty of the measurements is limited to only 1.75 times the Cramer-Rao lower bound and there are only two detectors to balance.

Table 1. Parameters of the Fe-Doppler lidar transmitter and receiver

\begin{tabular}{|r|l|}
\hline Transmitter & \\
\hline Power & $1.45 \mathrm{~W}$ \\
\hline Wavelength & $372.0995 \mathrm{~nm}$ \\
\hline Spectral width & $35-55 \mathrm{MHz}$ \\
\hline Rate & $35 \mathrm{~Hz}$ \\
\hline Pulse width & $250 \mathrm{~ns} \mathrm{FWHM}$ \\
\hline Divergence & $250 \mu \mathrm{rad}$ \\
\hline Receiver & \\
\hline FOV & $500 \mu \mathrm{rad}$ \\
\hline Aperture & $0.5 \mathrm{~m}^{2}$ \\
\hline PMT QE & $43 \%$ \\
\hline
\end{tabular}

A significant benefit of the Mach-Zehnder-receiverbased Fe Doppler lidar techniques described here is that all of the signal is recovered and no portion of the backscatter is discarded. Consequently, summation of the signals in both channels results in a quantity free of interference effects, provided the detectors are balanced. The same resonance Doppler lidar techniques for retrieving wind and temperature in the MLT region can be applied to the summation of signals from both channels. The Fe Doppler lidar with the multiple phase MZI techniques, by recovering the complete spectrum, enables a single lidar receiver to profile wind and temperature through the complete atmospheric column with nearly the same precision as the original instrument and without modification to any existing retrieval techniques. We believe that the successful implementation of such new technologies represents significant progress towards a whole atmosphere lidar that will enable new science avenues.

\section{ACKNOWLEDGEMENT}

We acknowledge the support of a NSF Major Research Instrumentation award (MRI-0723229), CRRL grant (AGS-1136272), a NASA Earth and Space Science Fellowship award (NNX09AO35H), and an internal CIRES Innovative Research Proposal award.

\section{REFERENCES}

[1] Chu, X. and G. C. Papen, 2005: Resonance Fluorescence Lidar for Measurements of the Middle and Upper Atmosphere, in Laser Remote Sensing, Fujii and Fukuchi, Eds. Taylor \& Francis Group, 179-432.

[2] Chu, X., Z. Yu, C. S. Gardner, C. Chen, and W. Fong, 2011: Lidar observations of neutral Fe layers and fast gravity waves in the thermosphere $(110-155 \mathrm{~km})$ at McMurdo (77.8 $\left.{ }^{\circ} \mathrm{S}, 166.7^{\circ} \mathrm{E}\right)$, Antarctica, Geophys. Res. Lett., 38, L23807, doi: 10.1029/2011GL050016.

[3] Chen, C., X. Chu, A. J. McDonald, S. L. Vadas, Z. $\mathrm{Yu}, \mathrm{W}$. Fong, and X. Lu, 2013: Inertia-gravity waves in Antarctica: A case study using simultaneous lidar and radar measurements at McMurdo/Scott Base, $J$. Geophys. Res. Atmos., 118, doi: 10.1002/jgrd.50318.

[4] Huang, W., X. Chu, B. Williams, S. Harrell, and C.-Y. She, 2009: Na double-edge magneto-optic filter for $\mathrm{Na}$ lidar profiling of wind and temperature in the lower atmosphere, Opt. Lett., 34(2), 199-201.

[5] Huang, W., X. Chu, J. Wiig, B. Tan, C. Yamashita, T. Yuan, J. Yue, S. D. Harrell, C.-Y. She, B. P. Williams, J. S. Friedman, and R. M. Hardesty, 2009: Field demonstration of simultaneous wind and temperature measurements from 5 to $50 \mathrm{~km}$ with a $\mathrm{Na}$ double-edge magneto-optic filter in a multi-frequency Doppler lidar, Opt. Lett., 34(10), 1552-1554.

[6] Liu, Z. and T. Kobayashi, 1996: Differential discrimination technique for incoherent Doppler lidar to measure atmospheric wind and backscatter ratio, Opt. Rev., 3(I), 47-52.

[7] Bruneau, D., 2001: Mach-Zehnder interferometer as a spectral analyzer for molecular Doppler wind lidar, Appl. Opt., 40(3), 391-399.

[8] Bruneau, D., A. Garnier, A. Hertzog, and J. Porteneuve, 2004: Wind-velocity lidar measurements by use of a Mach-Zehnder interferometer, comparison with a Fabry-Perot interferometer, Appl. Opt., 43(1), 173-182.

[9] Hilliard, R. and G. Shepherd, 1966: Wide-Angle Michelson Interferometer for Measuring Doppler Line Widths, J. Opt. Soc. Am., 56(3), 362-369.

[10] Smith, J. A., and X. Chu, 2015: High-efficiency receiver architecture for resonance-fluorescence and Doppler lidars, App. Opt., 54(13), in press.

[11] Chu, X., 2006: Alexandrite-ring-laser-based Fe Doppler lidar for mobile/airborne deployment, Proc. 23rd International Laser Radar Conference, 385-388.

[12] Hair, J., L. Caldwell, D. Krueger, and C.-Y. She, 2001: High-spectral-resolution lidar with iodine-vapor filters: measurement of atmospheric-state and aerosol profiles, Appl. Opt., 40(30), 5280-5294. 